Mitteilung des Verlags

15 Titeländerung: Transfusion Medicine AND HEMOTHERAPY

Editorial

17 Ein neuer Name, ein neues Profil - INFUSIOn Therapy and Transfusion Medicine WIRD ZU

Transfusion Medicine and Hemotherapy Sibrowski, W. (Münster)

6261 Pathogeninaktivierung von zellulären Blutbestandteilen - mehr oder weniger Sicherheit für den Patienten? Caspari, G.; Gerlich, W.H.; Gürtler, L. (Brandenburg)

Originalarbeiten

18 Eine neue Methode zur durchflusszytometrischen Zählung CD4-positiver T-Zellen in nicht lysierten Blutproben

Greve, B.; Cassens, U.; Westerberg, C.; Göhde jun., W.; Sibrowski, W.; Reichelt, D.; Göhde, W. (Münster)

114 Durchflusszytometrische Bestimmung der Überlebenszeit und der 24-Stunden-Wiederfindungsrate von transfundierten Erythrozyten Zeiler, T.; Müller, J.T.; Kretschmer, V. (Marburg)

120 Vergleich des fluoreszenzzytometrisch bestimmten Blutverlusts bei Hüftendoprothesen-Implantantion unter Allgemein- oder Spinalanästhesie Hartmann, B.; Junger, A.; Benson, M.; Lorson, S.; Jost, A.; Klein, T.; Langefeld, T:; Hempelmann, G. (Gießen)

257 Die Beteiligung humaner Blutplättchen an der antimikrobiellen Abwehr Klinger, M.H.F.; Ulbricht, T. (Lübeck)

266 Prävalenz der H36D- und C282-Mutationen im HFEGen bei 3015 Blutspendern in Südwestdeutschland König, D.; Mattler, S.; Eichler, H.; Klüter, H.; Bugert, P. (Mannheim)

272 Pharmakokinetik von Hydroxyethylstärke (70/0,5) nach Mehrfachinfusion

Lehmann, G. (Jena); Asskali, F.; Förster, H. (Frankfurt/M.)
3109 Vergleich zwischen vier Blutentnahmemöglichkeiten zur durchflusszytometrischen Analyse der Thrombozytenfunktion Scheinichen, D. (Hannover); Kleine, H.-D. (Rostock); Koksch, M. (Leipzig); Brandl, M.; Heine, J.; Elsner, H.-A.; Bornscheuer, A. (Hannover)

3117 Nachweis erythrozytärer Alloantikörper im Gelzentrifugationstest

Lehmann, R.; Kiefel, V. (Rostock)

4157 Transport von Erythrozytenkonzentraten und Gefrorenen Frischplasmen mit einem Rohrpostsystem Liebscher, K.; Bogner, I.; Dietzel, H.; Kätzel, R. (Leipzig)

4165 Der individuelle patientenabhängige Einfluss der Erythrozytenlyse-Reagenzien auf die Bestimmung von Leukozyten-Subpopulationen Göhde, W.; Cassens, U. (Münster); Lehman, L.G. (Douala); Traoré, Y. (Ouagadougou); Göhde jun., W.; Berkes, P.; Westerberg, C.; Greve, B. (Münster)

5210 Management der präoperativen Eigenblutspende bei künstlichem Hüftgelenksersatz

Möllmann, M.; von dem Berge, M.M.; Gurlit, S.; Fard, F.T.; Sibrowski, W. (Münster)

Übersichtsarbeiten

127 Diagnose der hereditären Hämochromatose mit nichtinvasiven Methoden

Nielsen, P.; Fischer, R.; Engelhardt, R.; Düllmann, J. (Hamburg)

3124 Standardisierung von Plasmaprodukten Seitz, R. (Langen)

4172 Thrombozytentransfusion: Je kälter desto besser? Hoffmeister, K.M.; Hartwig, J.H. (Boston, MA)

4179 Bedeutung der Creutzfeld-Jakob-Erkrankung und ihrer Variante für die Transfusionsmedizin Sachse, C.; Greinacher, A. (Greifswald)

5214 Anti-IgA-vermittelte anaphylaktische Transfusionsreaktionen Yu, H.; Sandler, S.G. (Washington DC)

5222 Ontogenie des Immunsystems Durandy, A. (Paris) 
6264 Biologische Funktionen katalytischer Antikörper Latry, V.; Bayry, J; Thorenoor, N.; Kazatchkine, M.D.; Kaveri, S.V.; Lacroix-Desmazes, S. (Paris)

6270 Autoimmunes lymphoproliferatives Syndrom: Eine Erberkrankung mit hoher Inzidenz autimmun vermittelter Zytopenien

Stronceck, D.; Dale, J.K.; Straus, S.E. (Bethesda, MD)

6277 Febrile und allergische Reaktionen nach Transfusion von Thrombozytenkonzentraten Hartwig, D. (Lübeck); Klüter, H. (Mannheim)

Fallbericht

3130 Transplantat-spezifische Antikörpertolernaz nach akuter Abstoßungsreaktion durch Immunadsorption und Behandlung mit 15-Desoxyspergualin Wernet, D.; Braun, N.; Schnaidt, M. (Tübingen)

4186 Apoplex nach hochdosierter intravenöser Gabe von Immunoglobulinen

Mohaupt, M.G.; Krueger, T.; Girardi, V.; Mansouri Taleghani, B. (Bern)

5228 Übertragung von Hepatitis-B-Virus 2 Monate vor einer Hepatitis-Oberflächen-Antigen-Positivität des Spenderbluts

Meisel, H.; Endres, A.S.; Walter, H.U. (Berlin); Wend, U.C.; Gerlich, W.H. (Gießen)

Qualitätsmanagement

278 Qualitätsmanagement in Hinblick auf den Gebrauch von Blutprodukten unter besonderer Berïcksichtigung des Selbstinspektionsprogramms - ein Bericht aus einem Universitätsklinikum

Von Heymann, C.; Pruß, A.; Kastrup, M.; Marz, S.; Braun, J.; Kiesewetter, H.; Kox, W.J.; Spies, C. (Berlin)

Klinische Information

137 Aufrechterhaltung der Blutversorgung während Krieg und Belagerung - Schlussfolgerungen aus den Erfahrungen in Sarajewo, Bosnien-Herzegowina Haracic, M.; Simmonds, S. (Sarajewo); Krausbauer, U. (Koblenz)

287 Gutachten über transfusionsrechtliche, arzneimittelrechtliche und arztrechtliche Verantwortungsabgrenzungsfragen im Zusammenhang mit der Gewinnung von Plasma zur Fraktionierung Hasskarl, H. (Ludwigshafen)

\section{Treponema pallidum}

Arbeitskreis Blut, Untergruppe «Bewertung

Blutassoziierter Krankheitserreger»

5232 Hämovigilanz in Deutschland - Berichte an das PaulEhrlich-Institut über Verdachtsfälle von Transfusionsreaktionen im Beobachtungszeitraum Januar 1995 bis Dezember 2002

Graul, A.; Heiden, M.; Gräf, K.; Keller-Stanislawski, B. (Langen)

\section{Hepatitis-C-Virus (HCV)}

Arbeitskreis Blut, Untergruppe «Bewertung Blutassoziierter Krankheitserreger»

Kongressbericht

4189 Bericht vom TSE-Meeting «Safety of Blood Components», Frankfurt/M., 21. März 2003 Roth, W.K. (Frankfurt/M.)

Briefe an die Herausgeber

140 Zu Hansen E, Altmeppen J: Proposal for Internal Quality Management in Intra- and Postoperative Blood Salvage. Infus Ther Transfus Med 2002;29:147-150

140 Antwort des Autors

141 Zu Hasskarl H: Transfusionsgesetz vom 1. Juli 1998 (BGBI. I, S. 1752): Bestellung eines nicht institutsangehörigen Transfusionsverantwortlichen - gutachterliche Stellungnahme. Infus Ther Transfus Med 2002; 29:331-337

3144 Epidemiologie von HIV, HBV und HCV und die Sicherheit von Blutprodukten. Zu Stark K, et al: Infections with HIV, HBV and HCV among blood donors in Germany 1998 and 1999. Infus Ther Transfus Med 2002;29:305-307 Gerlich, W.H. (Gießen); Caspari, G. (Brandenburg)

3145 Antwort der Autoren Stark, K.; Offergeld, R. (Berlin)

6284 Zu Roth WK: Bericht vom TSE-Meeting «Safety of Blood Components», Frankfurt/M., 21. März 2003. Transfus Med Hemother 2003;26:189-193 Zimmermann, R.; Ringwald, J.; Eckstein, R. (Erlangen)

6285 Antwort des Autors Roth, W.K. (Frankfurt/M.) 
6287 Validierung eines Rohrpostsystems zwischen zwei Krankenhäusern für den Transport von Erythrozytenkonzentraten und Blutbeuteln. Zu Liebscher et al: Transport of red blood cell concentrates and fresh frozen plasma in a pneumatic tube system. Transfus Med Hemother 2003;30:157-162

Petersen, D.; Przybyla, I.; Paulsen, M.; Albersmeyer, K.; Jacobsen, R.; Möller, M.; Petersen, F.; Arnold, K.; Vorwig, K.-H- (Flensburg)

Buchbesprechung

$\mathbf{1} 42,4194,5251,6307$

Gesellschaftsmitteilungen

4 195, 6309

Innovationen

6310

Industrieforum

143, 2 98, 3 146, 4 199, 5253,6312
Tagungen und Kongresse

1 47, 2 99, 3 149, 4 201, 5254,6313

\section{Erratum}

Abstracts

6289 14th Congress of the European Society for Haemapheresis and Haemotherapy, Prag, 10-13. September 2003 - Abstracts Guest-Editors: Blaha, M. (Hradec Králové); Gasova, Z. (Prague)

6316 Autorenverzeichnis $\mathbf{2 0 0 3}$

6318 Sachwortverzeichnis 2003

Supplement 1

36. Jahreskongress der Deutschen Gesellschaft für Transfusionsmedizin und Immunhämatologie e.V. Innsbruck, 16.-19. September 2003. Abstracts

Gast-Herausgeber: D. Schönitzer, Innsbruck 